\title{
VALIDAÇAO DE CONTEÚDO E APARÊNCIA DE UM CURSO ONLINE PARA A VIGILÂNCIA DA INFLUENZA
}

\author{
VALIDACIÓN DE CONTENIDO Y APARIENCIA DE UN CURSO ONLINE PARA \\ EL VIGILANCIA DE LA INFLUENZA
}

\author{
VALIDATION OF CONTENT AND APPEARANCE OF AN ONLINE COURSE \\ FOR THE SURVEILLANCE OF INFLUENZA
}

\author{
Andréa Soares Rocha da SILVA ${ }^{1}$ \\ Fabiane do Amaral GUBERT ${ }^{2}$ \\ Ivana Cristina Vieira de LIMA $^{3}$ \\ Raquel de Melo ROLIM ${ }^{4}$ \\ Diego Rodrigues TAVARES ${ }^{5}$ \\ Daiana Araújo da SILVA ${ }^{6}$ \\ Walquiria Aparecida Ferreira de ALMEIDA $^{7}$
}

RESUMO: No Brasil, as infecções respiratórias agudas causadas pela influenza são frequentes na população e demandam vigilância constante dos serviços de saúde, o que tem urgido novas tecnologias educacionais para os profissionais de saúde que trabalham na prevenção, promoção e tratamento da doença. O objetivo do estudo é validar o conteúdo e a aparência de um curso online, na modalidade a distância, para a vigilância da influenza, junto a juízes com experiência na rede de vigilância em saúde e epidemiológica. Trata-se de um estudo metodológico, a partir do referencial de Falkemback (2015) e cálculo do índice de validade de conteúdo (IVC). Após o desenvolvimento do curso foi realizada a validação, incluindo dos aspectos pedagógicos que envolveram a formação junto a 13 juízes especialistas nas áreas de educação a distância e vigilância em saúde e epidemiológica na área de influenza (conteúdo,) respectivamente; os mesmos analisaram os seguintes pontos: acessibilidade; usabilidade; funcionalidade; conteúdo; relevância; eficiência e ambiente. Segundo os resultados, os juízes eram na maioria do sexo feminino, oito eram enfermeiros e os demais atuavam na área de odontologia, biologia, farmácia, medicina e medicina

1 Universidade Federal do Ceará (UFC), Fortaleza - CE - Ceará, Brasil. 1Professora Adjunta IV, Departamento de Fisioterapia. Docente do Mestrado Profissional em Saúde da Família RENASF/UFC.

2 Universidade Federal do Ceará (UFC), Fortaleza - CE - Ceará, Brasil. Professora Adjunta III, Departamento de Enfermagem. Docente do Mestrado Profissional em Saúde da Família RENASF/UFC

${ }^{3}$ Universidade Federal do Ceará (UFC), Fortaleza - CE - Ceará, Brasil. Doutoranda do Programa de Pós Graduação em Enfermagem da Universidade Federal do Ceará, Bolsista CNPQ.

${ }^{4}$ Universidade Federal do Ceará (UFC), Fortaleza - CE - Ceará, Brasil. Gerente de Produção de Objetos de Aprendizagem do Núcleo de Tecnologia e Educação a Distância em Saúde da Universidade Federal do Ceará (NUTEDS/UFC).

${ }^{5}$ Universidade Federal do Ceará (UFC), Fortaleza - CE - Ceará, Brasil. Gerente Executiva da UNASUS/UFC e coordenadora de planejamento didático e de oferta de Módulos Educacionais para Cursos na modalidade a Distância.

${ }^{6}$ Enfermeira, Especialista em Especialista em Gestão de Sistemas e Serviços de Saúde; consultoria técnica em Influenza, pelo Ministério da Saúde- MS. Brasília, Distrito Federal, Brasil.

7 Enfermeira, Especialista em Especialista em Gestão de Sistemas e Serviços de Saúde; consultoria técnica em Influenza, pelo Ministério da Saúde- MS. Brasília, Distrito Federal, Brasil. 
veterinária; em relação ao IVC, este foi próximo a 1, evidenciando a pertinência e qualidade do curso online. Ao final, pode-se dizer que a formação, por meio de recursos pedagógicos e didáticos virtuais para profissionais de saúde de todo Brasil, é uma tecnologia educativa virtual que tem possibilitado a aprendizagem colaborativa facilitando o manejo da temática influenza.

PALAVRAS-CHAVE: Educação a distância. Vírus da influenza A. Estudos de validação. Educação profissional.

RESUMEN: En Brasil, las infecciones respiratorias agudas (IRA) causadas por la influenza son frecuentes en la población y demanda vigilancia constante de los servicios de salud, lo que ha urgido nuevas tecnologías educativas para el enfrentamiento de la enfermedad. En vista de ello, el objetivo del estudio es validar el contenido y la apariencia de un curso online para el vigilancia de la influenza, junto a jueces con experiencia en la red de vigilancia en salud y epidemiología. Se trata de un estudio metodológico, a partir del referencial de Falkemback (2015) y calculo del índice de validez de contenido (IVC). Después del desarrollo del curso se realizó la validación aparente, de contenido y de los aspectos pedagógicos que involucraron la formación, por jueces considerados especialistas en las áreas de educación a distancia $y$ vigilancia en salud y epidemiología en el área de influenza (contenido) respectivamente. En total, 13 jueces participaron en esta etapa de validación y analizaron los siguientes puntos: Accesibilidad; Usabilidad; Funcionalidad; Contenido; Relevancia; Eficiencia y Medio Ambiente. Según los resultados, los jueces eran en la mayoría del sexo femenino, ocho eran enfermeros y los demás actuaban en el área de odontología, biología, farmacia, medicina y medicina veterinaria y en relación al índice de validez de contenido hubo consenso entre los participantes. Al final se puede decir que el curso, por medio de recursos pedagógicos y didácticos específicos es una tecnología educativa virtual que ha posibilitado el aprendizaje colaborativo facilitando el aprendizaje sobre la temática influenza.

PALAVRAS CLAVE: Educación a distancia. Virus de la iInfluenza A. Estudios de validación.

ABSTRACT: In Brazil, acute respiratory infections (ARI) caused by influenza are frequent in the population and demand constant vigilance from the health services, which has urged new educational technologies for coping with the disease. In view of this, the objective of the study is to validate the content and appearance of an online course for the surveillance of influenza, together with judges with experience in the health surveillance and epidemiological network. It is a methodological study, based on Falkemback's (2015) reference and calculation of content validity index (CVI). After the development of the course, there was the apparent validation, content and pedagogical aspects that involved the training, by judges considered specialists in the areas of distance education and epidemiological and health surveillance in the area of influenza respectively. In total, 13 judges participated in this validation stage and analyzed the following points: Accessibility; Usability; functionality; Content; Relevance; Efficiency and Environment. According to the results, the judges were mostly female, eight were nurses and the other were in the area of dentistry, biology, pharmacy, medicine and veterinary medicine and in relation to the content validity index there was consensus 
among the participants. At the end, it can be said that the course, through specific pedagogical and didactic resources, is a virtual educational technology that has enabled collaborative learning to facilitate learning about influenza.

KEYWORDS: Education distance. Influenza A virus. Validation studies.

\section{Introdução}

A influenza é uma doença respiratória infecciosa de origem viral, que pode levar a complicações graves e ao óbito, especialmente nos grupos de alto risco para as complicações, como idosos, crianças e pessoas imunocomprometidas. De acordo com a Organização Mundial da Saúde, 5 a $15 \%$ da população pode vir a contraí-la no mundo, causando de 3 a 5 milhões de casos graves e de 250.000 a 500.000 mortes todos os anos (WHO, 2016).

No Brasil, as infecções respiratórias agudas (IRA) causadas pela influenza são frequentes na população e demandam vigilância constante dos serviços de saúde, o que tem urgido novas tecnologias educacionais em saúde. Para a vigilância da influenza, a capacitação dos profissionais de saúde deve assentar-se em contextos reais que envolvem o processo saúde-doença, a notificação das infecções, bem como sobre o acompanhamento dos casos e os conceitos de epidemiologia, adequando esses conhecimentos ao tempo e espaço, e principalmente em relação às características de distribuição das doenças nas diferentes regiões do país.

Diante do exposto e da possibilidade de construção de novas tecnologias para a educação na área da saúde, este artigo tem como intuito apresentar a experiência do Núcleo de Tecnologias e Educação a Distância em Saúde da Universidade Federal do Ceará - NUTEDS/UFC, na validação de um curso online acerca da temática influenza, a partir de demanda oriunda do Ministério da Saúde, junto a juízes com expertise na área, tendo como público-alvo profissionais das áreas de vigilância em saúde e epidemiológica de todo país.

O NUTEDS, criado pela portaria institucional $n^{\circ} 76$, de 15 de janeiro de 2010, tem com principais finalidades apoiar projetos no uso de Novas Tecnologias da Informação e da Comunicação (NTICs) na área da saúde, e atende todo o Ceará; entre as iniciativas apoiadas, destaca-se o Telessaúde e, mais recentemente, a parceria com a Universidade Aberta do SUS (UNASUS), ofertando cursos de educação a distância para todo o Brasil. 
Instituições de ensino têm produzido diversas experiências favoráveis ao uso das Tecnologias da Informação e Comunicação - TIC na formação dos profissionais da saúde (Carbonero, 2016), como objetos educacionais digitais e formações a distância que tem contribuído para ensino em diversas áreas, principalmente na Estratégia de Saúde da Família, realização de punção venosa (Frota et al 2015), vigilância das arboviroses para agentes comunitários (Silva et al 2016), curso de suporte básico de vida (Cavalcante et al 2016), entre outras iniciativas exitosas.

Para uma TIC ter aplicabilidade, principalmente na formação de recursos humanos, é necessário submetê-la a um processo de validação por juízes, técnica que pode ser utilizada para verificar a validade do conteúdo ou aparência de uma tecnologia (GUOHONG et al 2012). O conceito de validade é abordado como o grau em que um instrumento/proposta mostra-se apropriado para mensurar o que supostamente deveria medir ou alcançar. Assim, quando se submete um instrumento, e no caso deste estudo, um curso de educação a distancia, ao procedimento de validação, verifica-se se o objeto educacional proposto consegue alcançar seu objetivo.

Acrescenta-se a necessidade de análise dos processos de qualidade e eficácia das ações educacionais ofertadas para os profissionais de saúde de todo Brasil (Prado et al., 2012), os quais têm sido prioridade para as políticas de educação aberta. Entretanto, ainda são raros os estudos sistemáticos na área da educação sobre construção e validação de experiências educacionais.

Rangel et al (2012) consideram que, quando as atividades de ensino e aprendizagem são realizadas por meio de estratégias pedagógicas que valorizam os sujeitos e possuem excelência em sua concepção e elaboração, a intersubjetividade e a aprendizagem colaborativa tornam-se mais atrativas e satisfatórias para alunos/cursistas e professores/tutores envolvidos no processo.

Portanto, a validação do curso possibilita verificar o que não foi compreendido, o que deve ser acrescentado ou aperfeiçoado, além de se perceber algum distanciamento entre o que foi exposto e o que foi compreendido pela população-alvo. Frente ao exposto, o objetivo do estudo é validar o conteúdo e a aparência junto a juízes com experiência na rede de vigilância em saúde e epidemiológica no manejo da Influenza.

\section{Metodologia}


Trata-se de um estudo metodológico, que se propôs a validar o conteúdo e aparência de um Curso na modalidade de educação a distancia intitulado: Curso de capacitação sobre influenza para profissionais de vigilância em saúde na modalidade a distância online. O curso é dividido em três módulos e aborda aspectos relacionados à vigilância epidemiológica, à vigilância da influenza no Brasil, medidas de prevenção e controle, respostas rápidas em situação de surto e questões relacionadas ao atendimento e à assistência, tendo como público-alvo profissionais da saúde de todo o Brasil. No total, depois da validação, a expectativa é ofertar 1000 vagas.

O referencial teórico que permeou a elaboração do curso foram os pressupostos de (Falkembach, 2005), que comenta sobre o desenvolvimento de materiais educativos digitais a partir das seguintes etapas: Análise e Planejamento, Modelagem, Implementação, Avaliação e Distribuição. Neste estudo daremos ênfase para a Avaliação (validação por juízes).

Após o desenvolvimento do curso foi realizada a validação aparente, de conteúdo e dos aspectos pedagógicos que envolveram a formação, por juízes considerados especialistas nas áreas de educação a distância e vigilância em saúde e epidemiológica na área de influenza (conteúdo), respectivamente. Como critérios de inclusão para a definição dos juízes das áreas optou-se por realizar um edital de seleção para tutores com perfil de juízes, os quais deveriam possuir atuação acadêmica, docente ou prática na área de estudo, segundo recomendações adaptadas de Teles et al (2014).

A opção do edital divulgado a nível nacional foi captar o maior número de profissionais que realmente tivessem expertise prática e não somente teórica sobre o tema, visto que a influenza em si não é um tema que pode ser apreendido somente na academia, mas que demanda contato direto com as reais práticas de saúde e território vivo da saúde. Ao total, 13 usuários tutores participaram desta etapa de validação e analisaram os seguintes pontos: acessibilidade; usabilidade; funcionalidade; conteúdo; relevância; eficiência e ambiente (JOSEPH, 2012; EMORY UNIVERSITY, 2012).

A validação tem por objetivo avaliar o grau em que cada elemento do curso, neste caso, é relevante e representativo de um específico constructo com um propósito particular de avaliação, no qual os elementos são todos os aspectos do processo de medida que podem afetar a coleta de dados. No caso de um questionário, consideram-se como elementos as instruções, o formato das respostas e os itens de forma individual (ALEXANDRE, COLUCI, 2011). 
Destaca-se que o número de participantes do estudo seguiu recomendações de autores que aconselham um número impar de sujeitos/juízes para validação de tecnologias (LOBIONDO-WOOD, HABER, 2001). Além disso, foram verificadas variáveis sociodemográficas e educacionais dos participantes tais como sexo, idade, tempo de formação, experiência na área a fim de traçar o perfil dos juízes.

Para a etapa de validação foi calculado o Índice de Validade de Conteúdo (Content Validity Index) que avalia a concordância dos juízes quanto à representatividade da medida do conteúdo estudado. Por este método, os itens e instrumentos são considerados válidos se obtiverem um IVC de 0,80 (LOBIONDOWOOD, HABER, 2001). Foi realizada também análise qualitativa das sugestões e comentários dos juízes de modo a nortear as adequações necessárias para validação do Curso.

Este estudo foi aprovado pelo Comitê de Ética em Pesquisa da UFC sob o parecer $\mathrm{N}^{\mathrm{o}} 1.757 .613$, respeitando os princípios éticos para a pesquisa envolvendo seres humanos.

\section{Resultados e discussão}

Observando os participantes. a maioria era do sexo feminino, oito eram enfermeiros e os demais atuavam na área de odontologia, biologia, farmácia, medicina e medicina veterinária, totalizando 13 juízes. O tempo de graduação foi de 13 anos, e cerca de sete atuavam no Ministério da Saúde de diversas regiões do Brasil. Acerca da titulação quatro possuíam o titulo de mestre e todos atuavam em área de vigilância da influenza, há pelo menos um ano, com média de cinco anos. Um ponto importante é que 12 juízes já haviam participado de capacitações na área do estudo. Na tabela 1 são demonstrados os índices de concordância dos itens avaliados. Os dados estão apresentados na ordem do instrumento de avaliação. 
Tabela 1: Índice de Validade de Conteúdo (IVC) obtido pelos juízes tutores segundo os itens acessibilidade, usabilidade, funcionalidade, conteúdo, relevância e ambiente. Fortaleza/CE, jun, 2017.

\section{Questão da Avaliação}

\section{IVC}

\section{Acessibilidade}

1a. O curso de capacitação sobre influenza para profissionais de vigilância em saúde é de fácil acesso

1b. O ambiente virtual facilita a comunicação e interação entre os participantes (incluindo outros cursistas e tutor)

1c. O acesso aos recursos midiáticos (figuras/imagens, rádios, links, animações, jogos) é rápido

\section{Usabilidade}

2a. Fácil de entender as orientações de navegação, página a página, seção a seção, ou de um link para o outro, sem ficar perdido ou confuso adequada

2b. O ambiente permite navegar pelo conteúdo de forma forma clara

2c. O ambiente virtual de aprendizagem (AVA) fornece ajuda de

2d. A identidade/leiaute do curso é apresentado de forma atraente

\section{Funcionabilidade}

3a. O capacitação atende os objetivos propostos

3b. As ferramentas de comunicação com a gestão do curso ou 1,0 entre os próprios cursistas e/ou com o tutor estão implementadas de forma adequada

3c. O design do curso e recursos pedagógicos condizem com os

objetivos propostos

3d. A velocidade de execução dos recursos midiáticos é adequada

3e. A ambiente virtual apresenta design responsivo, se adequando bem a qualquer dispositivo de acesso (celular, tablet, computador)

\section{Conteúdos}

4a. Os conteúdos apresentados no curso contribuem para atuação dos profissionais nas ações de rotina e situações de emergência

4b. A estrutura didática e de conteúdo do curso é adequada

4c. O estilo de redação é fácil de ser compreendido

4d. O uso das imagens corresponde às informações do texto

\section{Relevância}

5a. Os recursos midiáticos (figuras/imagens, rádios, links, animações, jogos) facilitam o aprendizado sobre a temática em questão

5 b. A estrutura didática do curso contribui para o aprendizado dos conteúdos

5c. O material de apoio foi relevante para tirar duvidas, inclusive após a conclusão do curso.

5d. O curso estimulou a troca de informações sobre a temática com outros cursistas e/ou outros profissionais de saúde sobre o tema Influenza

6.Ambiente

6a. As páginas são adequadas para os tipos de informação que se apresentam

6b. O curso propõe diferentes situações de aprendizagem $\quad 1,0$

6c. Gostaria de continuar a utilizar o material do curso para estudar sobre a temática influenza 
No critério acessibilidade, que trata da facilidade de interação e comunicação, rapidez no acesso, incluindo as imagens que compunham a formação, o IVC alcançado nas respostas foi satisfatório evidenciando que o curso pode ser ofertado para profissionais com pouco letramento digital, já que sua interface é de fácil acesso. A Internet, com seus recursos, permite o acesso dos profissionais de saúde às novas tecnologias ampliando conhecimentos, informação e experiências, o que permite que profissionais em diferentes regiões tenham acesso igualitário à formação profissional (CAMARGO, ITO, 2012).

O ambiente virtual segundo os juízes facilitou a comunicação e interação entre os usuários e obteve IVC 1,0, evidenciando consenso entre os participantes. Para Moore e Kearley (2007), esse componente exerce impacto considerável sobre os demais elementos do sistema de um sistema de $\mathrm{EaD}$, e consiste do ambiente no qual os participantes de um curso a distância interagem entre si e com o material didático do curso. Gossi, Moraes e Brescia (2013) destacam ainda a necessidade de ferramentas de interação e comunicação nesses ambientes, a fim de que ocorra toda a veiculação das atividades a serem desenvolvidas em um curso online.

Segundo Mahmodi e Ebrahmzade (2015), os quais investigaram a relação entre interação instrucional e persistência do aluno em e-learning, as ferramentas de comunicação disponíveis nos cursos a distância podem facilitar o feedback e promover a interação entre os participantes, estando diretamente relacionadas também à persistência no curso, e recomendam ainda que se utilizem métodos diversos, pois uns podem ser mais atrativos do que outros em relação à persistência em cursos online.

Outro ponto relacionado é o acesso aos recursos midiáticos de forma rápida, fato que interfere na participação e motivação no cursista durante sua participação. Beluce e Oliveira (2015, p. 105) destacam a importância do "engajamento e autonomia do estudante" como aspectos importantes na motivação para a aprendizagem, e o foco de seu estudo recaiu na aprendizagem em ambientes virtuais. Frente a isso e diante de diversas tecnologias é importante que os conteudistas e elaboradores dos materiais reflitam sobre como e quando usá-las de forma mais efetiva.

Quanto à usabilidade que trata das facilidades de manejo, entendimento das informações, ajuda oferecida pelo curso, adequabilidade das cores e fontes, e se o ambiente permite navegar pelo conteúdo; o IVC em todas as respostas foi > 0,90, sendo, portanto, considerado satisfatório. A avaliação destes itens é de estrema importância, 
pois a qualidade do material e todo o conjunto fazem com o que o cursista tenha mais interesse em entrar no ambiente e usufruir do conteúdo ofertado.

Apesar de ter obtido consenso entre os juízes acerca das orientações de navegação sem deixar o cursista confuso, um dos juízes ponderou que este item poderia ser fortalecido, pois em alguns momentos sentiu-se perdido na navegação. Frente a este contexto é relevante a abordagem multidisciplinar na elaboração de um curso online, visto que a acessibilidade deve envolver as diversas peculiaridades dos participantes, seja de acordo com a faixa etária, letramento digital, experiências prévias com tecnologia, entre outras variáveis que podem influenciar a experiência no curso. Além disso, apesar da influenza, agravo objeto desta capacitação ter normas e condutas específicas nacionalmente, o contexto das regiões muitas vezes é diferente, o que pode causar diferentes percepções nos usuários.

Acerca da usabilidade é importante destacar que apesar do curso ser adequado para o fim o qual se destina, algumas singularidades devem ser reconhecidas. Em estudo de Rosial et al (2016) voltado para experiências de EAD na área de saúde no Brasil, dentre as dificuldades apontadas na pesquisa que mais sobressaíram foram formação de equipe técnica capacitada $(20,3 \%)$ e problemas com internet nos municípios $(16,4 \%)$ mencionadas por diretores de todas as regiões, seguidas por domínio de informática pelos cursistas $(12,7 \%)$ e necessidade de financiamento das instalações e aquisição de equipamentos $(12,6 \%)$. Esta realidade é parecida em várias regiões brasileiras e ainda são um impeditivo para a consolidação da EaD no país.

A funcionalidade trata do propósito do curso, se as funções de comunicação são implementadas, aparecimento na tela, design e velocidade são adequados. Neste critério, o item que tratou das ferramentas de comunicação também apresentou IVC elevado. A comunicação torna-se ferramenta essencial, pois o ambiente virtual pode ser considerado solitário em muitos momentos, e a interação por meio da comunicação com a coordenação do curso, tutores e suporte técnico são um ponto que fortalece o vínculo do cursista com a aprendizagem virtual.

No critério referente ao conteúdo, foram analisados se estes correspondem aos objetivos do curso, se são variados, bem estruturados, aparência diferente e agradável, e se o uso das imagens corresponde às informações do texto. Neste critério, todos os itens obtiveram IVC 1,0. Não há dúvidas de que a tecnologia está revolucionando a educação e que continuará a oferecer desafios e oportunidades; no entanto, apenas a tecnologia 
não melhora automaticamente o processo de aprendizagem. Pra ser bem-sucedida, o foco deve estar no ensino e aprendizagem, e não na tecnologia.

Para Silva et al (2015), o conhecimento da influenza no Brasil é essencial para continuarmos com a redução das taxas de infecção pelo vírus H1N1 que desde 2010 diminuíram, porém ainda é notório alguns casos e surtos da doença em território nacional. Para minimizar tais casos é importante, dentre outras medidas, a qualificação do trabalhador de saúde com conteúdos que contribuam para atuação dos profissionais nas ações de rotina e situações de emergência.

Na relevância do curso, foi avaliado se os recursos midiáticos ajudam a entender os aspectos-chave, se as divisões dos temas ajudam o entendimento, se o usuário retornou ao curso para tirar dúvidas e se conversou ou trocou ideias com demais cursistas sobre o material. Acerca destes recursos pedagógicos e didáticos específicos, sempre foi um fator singular a utilização de mídias entre os docentes, desde a Educação Básica até a formação superior, o que tem representado uma aproximação entre docentes e educandos. Moran (2012) ressalta que o uso de tecnologias amplia os horizontes de escolas e universidades, que se abre de forma a possibilitar que as pessoas se intercomuniquem, socializando informações, o que facilita a educação continuada através da integração entre mídias, com possibilidades síncronas e assíncronas, em horários favoráveis a cada indivíduo, facilitando até mesmo o contato entre educadores e educandos.

A ampliação das TIC por meio da convergência midiática facilita a interlocução entre diferentes recursos de mídias e esta realidade é importante, pois tais mudanças favorecem a aproximação dos saberes formais à realidade de vivências dos cursistas. Ou seja, toda essa evolução exige a formação de um tutor mediador e pesquisador reflexivo (SCHALCH; SANTOS, 2015).

\section{Considerações finais}

A realização deste estudo possibilitou o processo de construção e a validação de um curso online na modalidade a distância para profissionais de saúde de todas as regiõe do Brasil. O processo de validação da tecnologia educativa foi satisfatório com o IVC global próximo a 1, tornando, assim, o material validado dentro das normativas 
científicas do estudo metodológico. As sugestões dos juízes foram acatadas, buscando clareza e aplicabilidade do curso para o seu público-alvo.

Acredita-se que o processo de validação de tecnologias da informação e comunicação, por meio de cursos de educação a distância, por exempo, contribui cada vez mais para o fortalecimento das bases pedagógicas que envolvem o processo educativo formal de profissionais de saúde, objeto deste estudo. Assim, a educação profissional necessita buscar novas formas de interação que favoreçam a autonomia do cursista e que respeite suas necessidades de letramento digital e de conhecimento em saúde específico.

A temática em questão, voltada para o manejo da influenza, é altamente relevante em nosso país, e por isso experiências que busquem fortalecer as bases conceituais e pedagógicas devem ser valorizadas, por isso, o trabalho colaborativo, realizado neste estudo, promoveu a interação no espaço virtual proporcionado pelo curso.

\section{REFERÊNCIAS}

ALEXANDRE, N. M. C.; COLUCI, M. Z.O. Validade de conteúdo nos processos de construção e adaptação de instrumentos de medidas. Ciênc. saúde col. Rio de Janeiro, v. 16, n. 7, p. 3061-3068, 2011 . Disponível em <http://www.scielo.br/scielo.php?script=sci_arttext\&pid=S141381232011000800006\&lng=pt\&nrm=iso>. Acesso em: 22 ago. 2017.

BELUCE, A. C.; OLIVEIRA, K. L. Students. Motivation for Learning in Virtual Learning Environments. Paidéia (Ribeirão Preto), Ribeirão Preto, v. 25, n. 60, p. 105-113, 2015. Disponível em: $<$ http://www.scielo.br/scielo.php?script=sci_arttext\&pid=S0103863X2015000100105\&lng=en\&nrm=iso>. Acesso em: 20 ago. 2017.

CAMARGO, A. L; ITO, M. Utilização das tecnologias de informação e comunicação na área da saúde: uso das redes sociais pelos médicos. J. Health Inform. São Paulo. v. 4, n.4, p. 165-169, 2012.

CARBONERO, F.C. Análise das publicações nacionais sobre educação a distância na área da saúde. Associação Brasileira de Educação a Distância, Volume 15, n.1. p.5259 Disponível em <http://seer.abed.net.br/edicoes/2016/04_Analise_das_publicacoes.pdf >. Acesso em: 05 jun. 2017.

DA SILVA F. R. et al. Produção e execução de um curso a distância como resposta rápida à epidemias emergentes: $\mathrm{O}$ caso do combate ao Mosquito Aedes Aegypti. Clin Biomed Res, Porto Alegre. 36(Supl), 2016. Disponível em: < 
http://hdl.handle.net/10183/149415>. Acesso em: 24 jul. 2017.

Disponível em: <http://www.cyberbee.com/guides_sites.html>. Acesso em: 20 Maio 2017.

FALEIRO F.R. G. et al. Educação a Distância e formação técnica em saúde: percepção dos Diretores das escolas técnicas do Sus no Brasil. Atas - Invest. Qual. Saúd. Porto, Portuga, .v.1. Disponível em:

<http://Proceedings.Ciaiq.Org/Index.Php/Ciaiq2016/Article/Viewfile/800/786>. Acesso em: 23.ago. 2017.

FALKEMBACH, G. A. M. Concepção e Desenvolvimento de Material Educativo Digital. Revista RENOTE. Porto Alegre, v.3, n1, p.1-15.2005. Disponível em: http://seer.ufrgs.br/renote/article/view/13742/7970. Acesso em: 20 jul. 2017.

FROTA, N. M. et al. Validation of educational hypermedia about peripheral venipuncture. Texto contexto - enferm., Florianópolis , v. 24, n. 2, p. 353-361, 2015. Disponível em: <http://www.scielo.br/scielo.php?script=sci_arttext\&pid=S0104$07072015000200353 \& \operatorname{lng}=e n \& n r m=i s o>$. Acesso em: 20 jul. 2017.

GROSSI, M. G. R.; MORAES, A. L.; BRESCIA, A. T. Interatividade em Ambientes Virtuais de Aprendizagem no processo de ensino aprendizagem na Educaçao a Distância. Rev. @ Rq. Bras. De Educação, Belo Horizonte, v. 1, n. 1, p. 75-92, 2013. Disponível em:

http://periodicos.pucminas.br/index.php/arquivobrasileiroeducacao/article/view/P.23187344.2013v1n1p75/5543. Acesso em: 28 jul.2017.

GUOHONG, G. et al. The Study on the Development of Internet-based Distance Education and Problems. Energy Procedia, v. 17, p.1362-1368. 2012. Disponível em: <https://doi.org/10.1016/j.egypro.2012.02.253>. Acesso em 05 jun. 2017.

JOSEPH, L. Cyberguide Ratings For Content Evaluation. Cyberguides, 2012.

LOBIONDO-WOOD, G.; HABER, J. Pesquisa Em Enfermagem: Métodos, Avaliação crítica e Utilização. 4. Ed. Rio De Janeiro: Guanabara Koogan, 2001.

MAHMODI, M., EBRAHIMZADE, I. The analysis of Iranian students' persistence in online education. The International Review of Research in Open and Distributed Learning, 16(1). 2015. Disponível em: <https://doi.org/10.19173/irrodl.v16i1.1982>. Acesso em 05 jun. 2017.

MASETTO, M. T. Mediação pedagógica e o uso de tecnologia: In: BEHRENS, M.A; MORAN, J.M. Novas tecnologias e mediação pedagógica. 19.ed. Campinas: Papirus, 2012.

MOORE, M. G. Educação a Distância: Uma Visão Integrada. In: MOORE, M. G., KEARSKEY, G. São Paulo: Thomson Learning, 2007.

PRADO, C. et al. Ambiente Virtual de Aprendizagem no Ensino de Enfermagem: Relato de Experiência. Rev. bras. enferm., Brasília , v. 65, n. 5, p. 862-866, 2012. Disponível em: <http://www.scielo.br/scielo.php?script=sci_arttext\&pid=S0034- 
71672012000500022\&lng=en\&nrm=iso>. Acesso em: 23 Aug. 2017

RANGEL-S, M. L. et al. Redes De Aprendizagem Colaborativa: Contribuição Da Educação a Distância no processo de qualificação de gestores do Sistema Único de Saúde - Sus. Interface (Botucatu), Botucatu , v. 16, n. 41, p. 545-556, 2012. Disponível em: <http://www.scielo.br/scielo.php?script=sci_arttext\&pid=S141432832012000200019\&lng=en\&nrm=iso>. Acesso em: 23 Aug. 2017.

SCHALCH, C. S; SANTOS, E. U. A utilização de recursos de mídias no processo de ensino aprendizagem: identificação de novos perfis docentes. Revista Educ. \& tec. Curitiba. n.15, p. 18-33, 2015. Disponível em:

http://revistas.utfpr.edu.br/pb/index.php/revedutec-ct/article/view/1921/1601. Acesso em: 15 jul. 2017.

SILVA, J. K. et al. A importância do conhecimento quanto ao vírus da influenza A (H1N1): relato de experiência. Rev. de Epidemiol e Control de Infec. Santa Cruz do Sul, v. 5, n. 4, p. 209-211, 2015. ISSN 2238-3360. Disponível em:

<https://online.unisc.br/seer/index.php/epidemiologia/article/view/6192>. Acesso em: 22 ago. 2017.

TELES, L. M. R. et al . Development and validating an educational booklet for childbirth companions. Rev. esc. enferm. USP, São Paulo , v. 48, n. 6, p. 977-984, 2014. Disponível em:

$<$ http://www.scielo.br/scielo.php?script=sci_arttext\&pid=S0080-

62342014000600977\&lng=en\&nrm=iso>. Acesso em: 22 Aug. 2017.

\section{Como referenciar este artigo}

SILVA, Andréa Soares Rocha da, et al. Validação de conteúdo e aparência de um curso online para vigilância da influenza. Revista Ibero-Americana de Estudos em Educação, Araraquara, v. 12, n. esp. 2, p. 1408-1420, ago./2017. Disponível em: <http://dx.doi.org/10.21723/riaee.v12.n.esp.2.10065>. E-ISSN: 1982-5587.

Submetido em: 08/07/2017

Aprovação final em: 18/08/2017 